Proc. Indian Acad. Sci. (Chem. Sci.), Vol. 1(M). No. 4, August 1988, pp. 353-357

(C) Printed in India.

\title{
Dynamics of polar solvation : Route to single exponential relaxation via translational diffusion ${ }^{\dagger}$
}

\author{
BIMAN BAGCHI* and AMALENDU CHANDRA \\ Solid State and Structural Chemistry Unit. Indian Institute of Science, \\ Bangalore 560012, India \\ MS received 25 June 1988
}

\begin{abstract}
A microscopic theoretical calculation of time-dependent solvation energy shows that the solvation of an ion or a dipole is dominated by a single relaxation time if the translational contribution to relaxation is significant.
\end{abstract}

Keywords. Polar solvation; single exponential relaxation: translational diffusion; timedependent solvation energy.

The dynamics of polar solvation is currently a subject of tremendous theoretical and experimental interest (Calef and Wolynes 1983; Bagchi et al 1984, 1988; Castner et al 1987, 1988; Maroncelli and Fleming 1987; Nagarajan et al 1987; Su and Simon 1987; Wolynes 1987; Nichols and Calef 1988; Rips et al 1988). Many recent experiments on solvation of a newly created charge or a dipole have measured the solvation time correlation function, $C_{s}(t)$, defined by

$$
C_{s}(t)=\frac{\bar{\nu}(t)-\bar{\nu}(\infty)}{\bar{\nu}(o)-\bar{\nu}(\infty)},
$$

where the $\bar{\nu}$ 's are the average frequencies of the emission spectra at various times. Experimental results reveal a rich and diverse behaviour of $C_{s}(t)$. The correlation function is single exponential in some polar liquids but markedly non-exponential in some others (Castner et al 1987; Maroncelli and Fleming 1987). If one defines an average 'experimental' relaxation time $\tau_{s}$ by

$$
\tau_{s}=\int_{0}^{\infty} \mathrm{d} t C_{s}(t),
$$

then $\tau_{s}$ usually falls in between the Debye relaxation time, $\tau_{D}$, of the polar solvent and the longitudinal relaxation time $\tau_{L}\left[=\left(\varepsilon_{x} / \varepsilon_{0}\right) \cdot \tau_{D}\right.$, where $\varepsilon_{\infty}$ and $\varepsilon_{0}$ are respectively the infinite and zero frequency dielectric constants of the polar solvent]. As $\varepsilon_{0}$ increases, $\tau_{s}$ approaches $\tau_{D}$. However, in some cases, like the

\footnotetext{
${ }^{+}$Contribution No. 545 from the Solid State and Structural Chemistry Unit

* For correspondence
} 
solvation of excited LDS 50 in methanol and butanol, $\tau_{s}$ is substantially smaller than $\tau_{L}$. This last observation has created some confusion and led to the suggestion that translational motion of the solvent molecules is important in this case. Several theoretical studies have been carried out to understand the experimental results.

In this work we present the rather surprising result that the /translational contribution can not only accelerate the solvation process, but it may also render the process single exponential in situations where the rotational contribution proceeds on several relaxation time scales.

Our analysis is based on the following Smoluchowski-Vlasov equation (SVE) for the position and orientation dependent density, $\rho(\mathbf{r}, \omega, t)$, of the solvent molecules

$$
\begin{aligned}
\frac{\partial}{\partial t} \delta \rho(\mathbf{r}, \omega, t) & =D_{R} \nabla_{\omega}^{2} \delta \rho(\mathbf{r}, \omega, t)+D_{T} \nabla^{2} \delta \rho(\mathbf{r}, \omega, t) \\
& -D_{R}\left(\frac{\rho_{0}}{4 \pi}\right) \nabla_{\omega}^{2} \int \mathrm{d} \mathbf{r}^{\prime} \mathrm{d} \omega^{\prime} c\left(\mathbf{r}, \omega, \mathbf{r}^{\prime}, \omega^{\prime}\right) \delta \rho\left(\mathbf{r}^{\prime}, \omega^{\prime}, t\right) \\
& -D_{T}\left(\frac{\rho_{0}}{4 \pi}\right) \nabla^{2} \int \mathrm{d} \mathbf{r}^{\prime} \mathrm{d} \omega^{\prime} c\left(\mathbf{r}, \omega, \mathbf{r}^{\prime}, \omega^{\prime}\right) \delta \rho\left(\mathbf{r}^{\prime}, \omega^{\prime}, t\right),
\end{aligned}
$$

where,

$$
\delta \rho(\mathbf{r}, \omega, t)=\rho(\mathbf{r}, \omega, t)-\rho_{\mathrm{eq}}(\mathbf{r}, \omega)=\rho(\mathbf{r}, \omega, t)-\rho_{0} / 4 \pi,
$$

where $D_{R}$ and $D_{T}$ are, respectively, the rotational and translational diffusion coefficients of the solvent molecules, $\rho_{0}$ is the equilibrium solvent density, $\nabla$ and $\nabla_{\omega}$ are, respectively, the spatial and the angular gradient operators, and $c\left(r, \omega, r^{\prime}, \omega^{\prime}\right)$ is the two-particle direct correlation function (Gray and Gubbins 1984). Equation 3 is strictly valid for a homogeneous isotropic dense liquid. It is derived on the assumption that spatial and angular momenta relaxations are very fast and that inertial effects can be neglected. The polarization fluctuation is given by

$$
\delta \mathbf{P}(\mathbf{r}, t)=\int \mathrm{d} \omega \hat{\alpha}(\omega) \delta \rho(\mathbf{r}, \omega, t),
$$

where $\hat{\alpha}(\omega)$ is a unit vector with the orientation given by $\omega$. The time dependent solvation energy is given by

$$
\Delta E(t)=\int \mathrm{d} k \mathbf{E}_{u}(\mathbf{k}) \cdot \delta \mathbf{P}(\mathbf{k}, t),
$$

where $\mathbf{E}_{0}(\mathbf{k})$ is the Fourier transform of the bare field of the ion or the dipole whose solvation is being investigated. In writing (5) we have assumed that the ion or the dipole perturbs the solvent only to a small degree so that $\operatorname{SP}(\mathbf{k}, t)$ can be given by (4) and (3).

Next, we evaluate $\delta P(k, t)$. This is done by substituting (4) in (3) and carrying out the appropriate integrations. We then perform a Fourier transform on the resulting expression to get (Chandra and Bagchi 1988)

$$
\begin{aligned}
\frac{\partial}{\partial t} \delta \mathbf{P}(\mathbf{k}, t)= & -\left(2 D_{R}+D_{T} k^{2}\right) \delta \mathbf{P}(\mathbf{k}, t) \\
& +\frac{\rho_{t}}{3}\left(2 D_{R}+D_{T} k^{2}\right) C(k) \cdot \delta \mathbf{P}(\mathbf{k}, t),
\end{aligned}
$$


where the tensor $C(k)$ is defined by (Gray and Gubbins 1984)

$$
c\left(k, \omega, \omega^{\prime}\right)=c_{\text {iso }}(k)+\hat{\alpha}(\omega) \cdot C(k) \cdot \hat{\alpha}\left(\omega^{\prime}\right)+\ldots
$$

We shall assume that $C(k)$ is given by the mean spherical approximation (MSA) theory; the conclusions presented here, however, do not depend critically on this assumption. If we divide $\delta \mathbf{P}(\mathbf{k}, t)$ into the usual longitudinal $\left(P_{L}\right)$ and transverse $\left(P_{T}\right)$ components, then (6) can be solved to obtain

$$
\begin{aligned}
& P_{L}(\mathbf{k}, t)=P_{L}(\mathbf{k}, o) \exp \left(-t / \tau_{L}(k)\right), \\
& P_{T}(\mathbf{k}, t)=P_{T}(\mathbf{k}, o) \exp \left(-t / \tau_{T}(k)\right),
\end{aligned}
$$

with

$$
\begin{aligned}
& \tau_{L}(k)=\left(2 D_{R}\right)^{-1}\left[1+p^{\prime}(k \sigma)^{2}-\frac{\rho_{0}}{3}\left(1+p^{\prime}(k \sigma)^{2}\right)\left(c_{\Delta}+2 c_{D}\right)\right]^{-1} \\
& \tau_{T}(k)=\left(2 D_{R}\right)^{-1}\left[1+p^{\prime}(k \sigma)^{2}-\frac{\rho_{0}}{3}\left(1+p^{\prime}(k \sigma)^{2}\right)\left(c_{\Delta}-c_{D}\right)\right]^{-1}
\end{aligned}
$$

where $p^{\prime}=D_{T} / 2 D_{R} \sigma^{2}, \sigma$ is the molecular diameter of a solvent molecule. $C_{\Delta}$ and $C_{D}$ are the usual MSA anisotropy functions (Gray and Gubbins 1984). The parameter $p^{\prime}$ is related to the van der Zwan-Hynes (1983) parameter $p$ by $p^{\prime}=p(a / \sigma)^{2}$ where $a$ is the diameter of the solute. In figure 1 , we have plotted

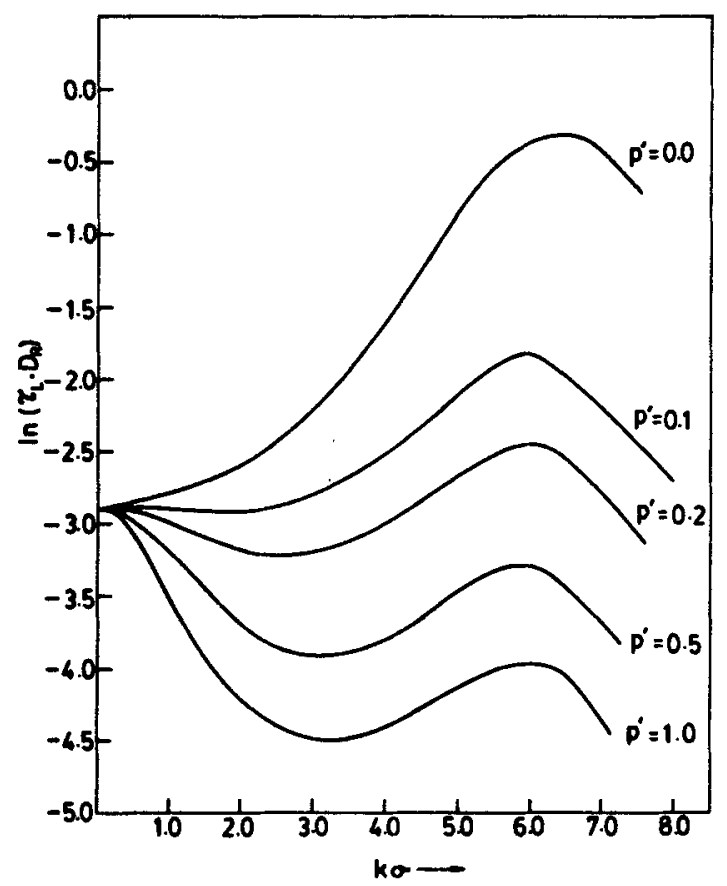

Figure 1. The longitudinal relaxation time $\tau_{l}(k)$ is plotted against the wave vector $k$ for several values of the parameter $p^{\prime}$. The other parameter values are: $\rho_{0}=0 \cdot 8, \varepsilon_{01}=30$. 
$\tau_{L}(k)$ against $k$ for several values of the parameter $p^{\prime}$. As can be seen from the graph, there are two distinct regions in the dependence of $\tau_{L}(k)$ on $k$. Near $k=0$, the dependence of $\tau_{L}(k)$ on $k$ is weak, especially so far $p^{\prime}<0 \cdot 2$. Then, $\tau_{L}(k)$ increases sharply, followed by a broad maximum at $k_{M}$ which is near $k=6 \cdot 2 / \sigma$. It is interesting to note that $k \sigma=6.2$ corresponds, in the real space, to the nearest neighbour distance. This maximum reflects the slow structural relaxation in dense liquids. For small $p^{\prime}$, we can write (5) in the following form

$$
\begin{aligned}
\Delta E(t) & =\left(\int_{k<k_{1}} \mathrm{dk} E_{0}(\mathbf{k}) P_{L}(k, 0)\right) \exp \left(-t / \tau_{L}\right) \\
& +\left(\int_{k_{1}>k>k_{1}} \mathrm{~d} \mathbf{k} E_{0}(\mathbf{k}) P_{L}(k, 0)\right) \exp \left(-t / \tau_{M}\right),
\end{aligned}
$$

where $k_{1}$ is a value between zero and $k_{M}$. If $\tau_{L}(k)$ is weakly varying near the origin, then the precise value of $k_{1}$ is not important. In most cases the first term in (10) is the dominant one. $k_{c}$ is a cut-off value which arises because of the molecular nature of the solute particle. Equation (10) approximately includes the two essential aspects of $\tau_{L}(k)$ for small $p^{\prime}(<0 \cdot 2)$.

Note that the division in (10) is similar to the one assumed by Wolynes (1987) in his approximate solution of the dynamic MSA. Equation (10) has a similar interpretation too. Note also that $\tau_{M} / \tau_{L}<\varepsilon_{0}$ and that $\tau_{M}$ is not identical with $\tau_{D}$. $\tau_{D}$ is given by the $k \rightarrow 0$ limit of $\tau_{T}(k)$ which is given by (9a).

The biexponential (or the multi-exponential in the exact treatment) nature of $\Delta E(t)$ disappears as $p^{\prime}$ is increased further. As can be seen from figure 1 , as $p^{\prime}$ becomes greater than half, $\tau_{M}$ gets substantially smaller than $\tau_{L}$. This behabiour is depicted in figure 2 where the ratio $\tau_{M} / \tau_{L}$ is plotted against $p^{\prime}$. For large $p^{\prime}$,

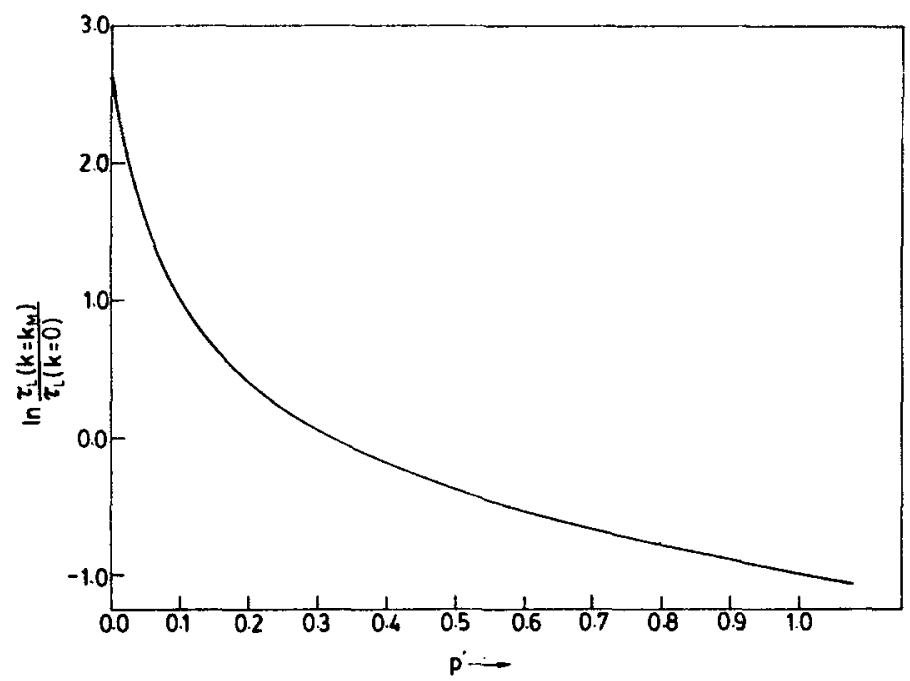

Figure 2. The ratio $\tau_{l}\left(k=k_{M}\right) / \tau_{l}(k=0)$ is plotted against the parameter $p^{\prime}$. As $p^{\prime}$ increases, the relaxation time constant $\tau_{,}\left(k=k_{M}\right)$ decreases rapidly. The other parameter values are the same as in figure 1. 
Table 1. Solvent properties at $20^{\circ} \mathrm{C}^{*}$.

\begin{tabular}{llcc}
\hline Solvent & $p$ & $\begin{array}{c}\tau_{L}=\left(\varepsilon_{x_{x}} / \varepsilon_{0}\right) \tau_{D} \\
(\mathrm{ps})\end{array}$ & $\begin{array}{c}\tau_{1} \\
(\mathrm{ps})\end{array}$ \\
\hline Acetonitrile & $0 \cdot 10$ & $0 \cdot 2$ & $0 \cdot 4$ \\
DMSO & $0 \cdot 10$ & $2 \cdot 1$ & $3 \cdot 1$ \\
Nitrobenzene & $0 \cdot 18$ & $5 \cdot 1$ & $3 \cdot 4$ \\
Methanol & $0 \cdot 76$ & $9 \cdot 2$ & $3 \cdot 3$ \\
$n$-Butanol & $1 \cdot 6$ & 120 & 66 \\
\hline
\end{tabular}

*from Castner (1988)

the long time dynamics will be dominated by behaviour near $k=0$; the rest will show up only as transients. In order to compare with experiments, we have collected the values of the parameter $p$ in table 1 taken from Castner (1988) for LDS-50 in several polar solvents. Note that $p^{\prime}=p(a / \sigma)^{2}$, where $a$ and $\sigma$ are the diameters of the solute and the solvent molecules respectively. Our theory predicts that for methanol and butanol, translational effects will wash out nonexponential behaviour, except perhaps in very short time periods. In these solvents, we predict that long time decay will be given by $\tau_{L}(k=0)$. This is drastically different from solvents with small $p$ value where the long time behaviour will be dominated by $\tau_{L}\left(k=k_{M}\right)$. We predict that for solvents with large $p$ values the continuum treatment of polarization relaxation is misleading, especially so if the intermediate to large values of the wave vector are probed by the solute. Such situations may arise in electron transfer reactions and also in the solvation of a dipole, as observed earlier.

We end this communication with a note of caution. The present calculations are based on MSA representation of the direct correlation function which is only-a crude representation of a real polar liquid, especially if it is protic and hydrogen-bonded. However, the translational effects predicted here are expected to be partially present even in such a solvent.

\section{References}

Bagchi B, Oxtoby D W and Fleming G R 1984 Chem. Phys. 86257

Bagchi B. Castner E W and Fleming G R $1988 \mathrm{~J}$. Mol. Struc. (in press)

Calef D F and Wolynes P G $1983 \mathrm{~J}$. Chem. Phys. 784145

Castner E W 1988 Ph.D. thesis, University of Chicago (unpublished)

Castner E W, Fleming G R and Bagchi B 1988a Chem. Phys. Lett. 143270

Castner E W, Fleming G R, Bagchi B and Maroncelli M 1988b J. Chem. Phys. (in press)

Castner E W, Maroncelli M and Fleming G R 1987 J. Chem. Phys. 861090

Chandra A and Bagchi B 1988 Chem. Phys. Lett. in press

Gray C G and Gubbins K E 1984 Theory of molecular fluids (Oxford: Clarendon) vol. 1

Maroncelli M and Fleming G R $1987 \mathrm{~J}$. Chem. Phys. 866221

Nagarajan V, Brearley A M, Kang T-J and Barbara P F 1987 J. Chem. Phys. 863183

Nichols A L and Calef D F $1988 \mathrm{~J}$. Chem. Phys. (in press)

Rips I, Klafter J and Jortner J 1988 J. Chem. Phys. 883246

Su S G and Simon J D 1987 J. Phys. Chem. 912693

van der Zwan G and Hynes J T 1983 Physica A121 227

Wolynes P G 1987 J. Chem. Phys. 865133 